\title{
Erratum: Topological Magnon Bands and Unconventional Superconductivity in Pyrochlore Iridate Thin Films \\ [Phys. Rev. Lett. 118, 177201 (2017)]
}

\author{
Pontus Laurell and Gregory A. Fiete
}

(a) (Received 21 May 2018; published 6 July 2018)

DOI: 10.1103/PhysRevLett.121.019901

First, while extending our magnon thermal Hall calculations to other systems, we noticed a missing factor of $1 /(2 \pi)^{2}$. Explicitly, in Eq. (18) of the Supplemental Material in the original Letter, $V=1$ was used instead of $V=(2 \pi)^{2}$. When this factor is accounted for, we find that the magnitude of the initial peak for triangular-kagome-triangular (TKT) systems with $U=2.5 \mathrm{eV}, t=U / 6$, is $2.2 \times 10^{-11} \mathrm{~W} / \mathrm{K}$ for $\theta=1.15$, and $1.7 \times 10^{-11} \mathrm{~W} / \mathrm{K}$ for $\theta=1.57$. For the kagome-triangular (KT) bilayer with $J=27.3 \mathrm{meV}$, we find $1.9 \times 10^{-12} \mathrm{~W} / \mathrm{K}$. Hence a sensitivity of $\lesssim 10^{-11} \mathrm{~W} / \mathrm{K}$ is required to resolve the sign change, rather than $\approx 10^{-10} \mathrm{~W} / \mathrm{K}$ as we initially reported. The Supplemental Material of the original Letter has been updated accordingly, and an updated version of Fig. 4 of the Letter is provided in this Erratum. This correction highlights the challenge of experimentally observing the effect in thin film systems, but does not affect the main conclusions of our Letter.

Second, we have corrected Eqs. (14)-(15) in the Supplemental Material of the original Letter, and added Eq. (16). This change addresses a typo, and has no effect on our results.

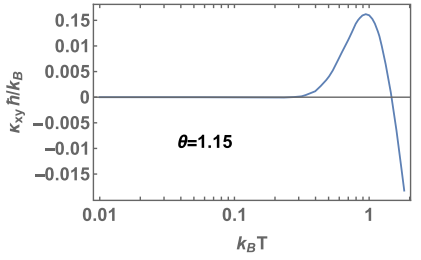

(a) TKT

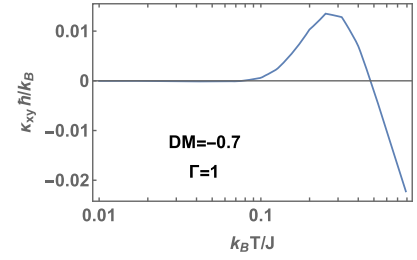

(b) KT

FIG. 4. Temperature dependence of the magnon Hall conductivity, $\kappa_{x y}(T)$ for (a) the TKT trilayer system and (b) the KT bilayer system, up to the estimated ordering temperature (see Supplemental Material in the original Letter). The sign change of the thermal Hall conductivity with temperature is associated with a change in which topological bands dominate the transport (see Supplemental Material in the original Letter). In (a) $k_{B} T$ is given in units of $t^{2} / U$. 